\title{
A selective oxidation on metallic carbon nanotubes by halogen oxoanions
}

Seon-Mi Yoon, ${ }^{\S}$ Sung Jin Kim, ${ }^{\ddagger}$ Hyeon-Jin Shin, ${ }^{\S}$ Anass Benayad, ${ }^{\S}$ Seong Jae Choi, ${ }^{\S}$ Ki Kang Kim, ${ }^{\ddagger}$ Soo Min Kim, ${ }^{\ddagger}$ Yong Jin Park, ${ }^{\ddagger}$ Gunn Kim, ${ }^{\ddagger}$ Jae-Young Choi, ${ }^{,},{ }^{\S}$ and Young Hee Lee ${ }^{*},{ }^{\ddagger}$

${ }^{\S}$ Display Device \& Material Lab, Analytical Engineering Center, Samsung Advanced Institute of Technology, P.O. Box 111, Suwon 440-600, Korea

${ }^{\ddagger}$ Department of Nanoscience and Nanotechnology, Department of Physics, and Center for Nanotubes and Nanostructured Composites, Sungkyunkwan Advanced Institute of Nanotechnology, Sungkyunkwan University, Suwon 440-746, Korea
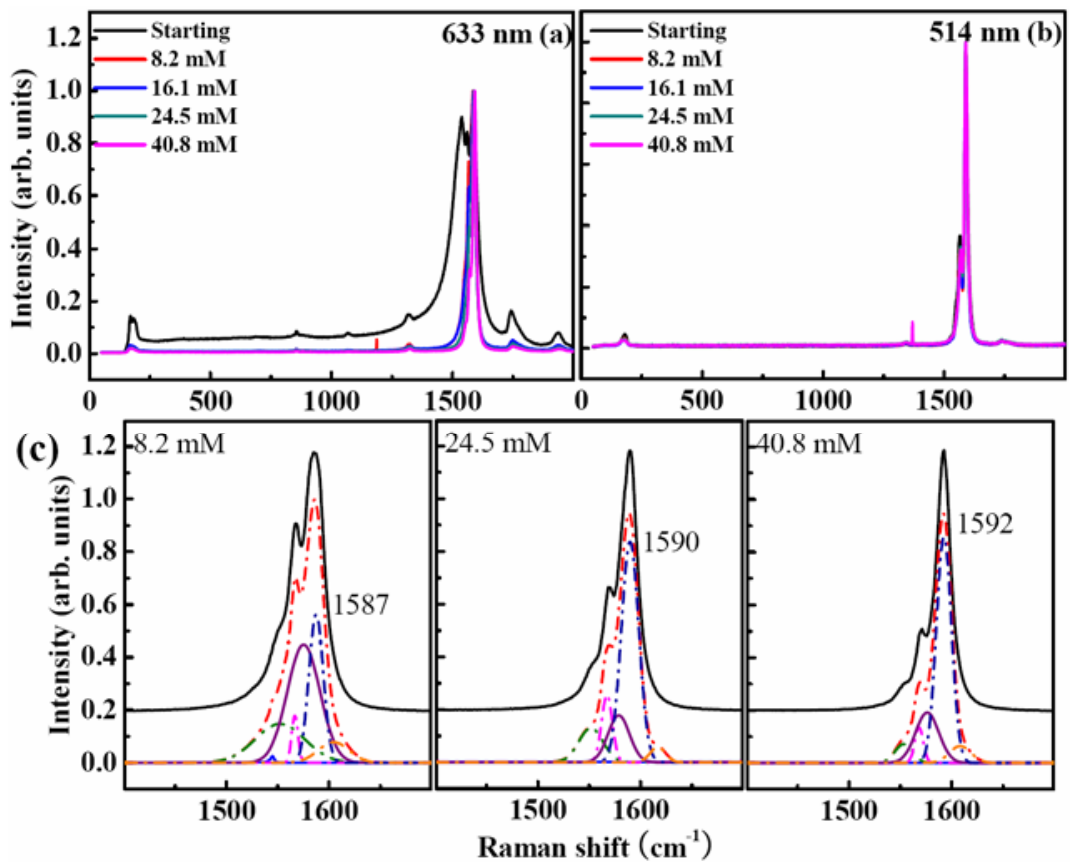

Figure S1: Raman spectra at an excitation of $633 \mathrm{~nm}$ and $514 \mathrm{~nm}$ of the pristine and chloro oxoanion treated samples at different concentration of $\mathrm{NaClO}_{4}$. 


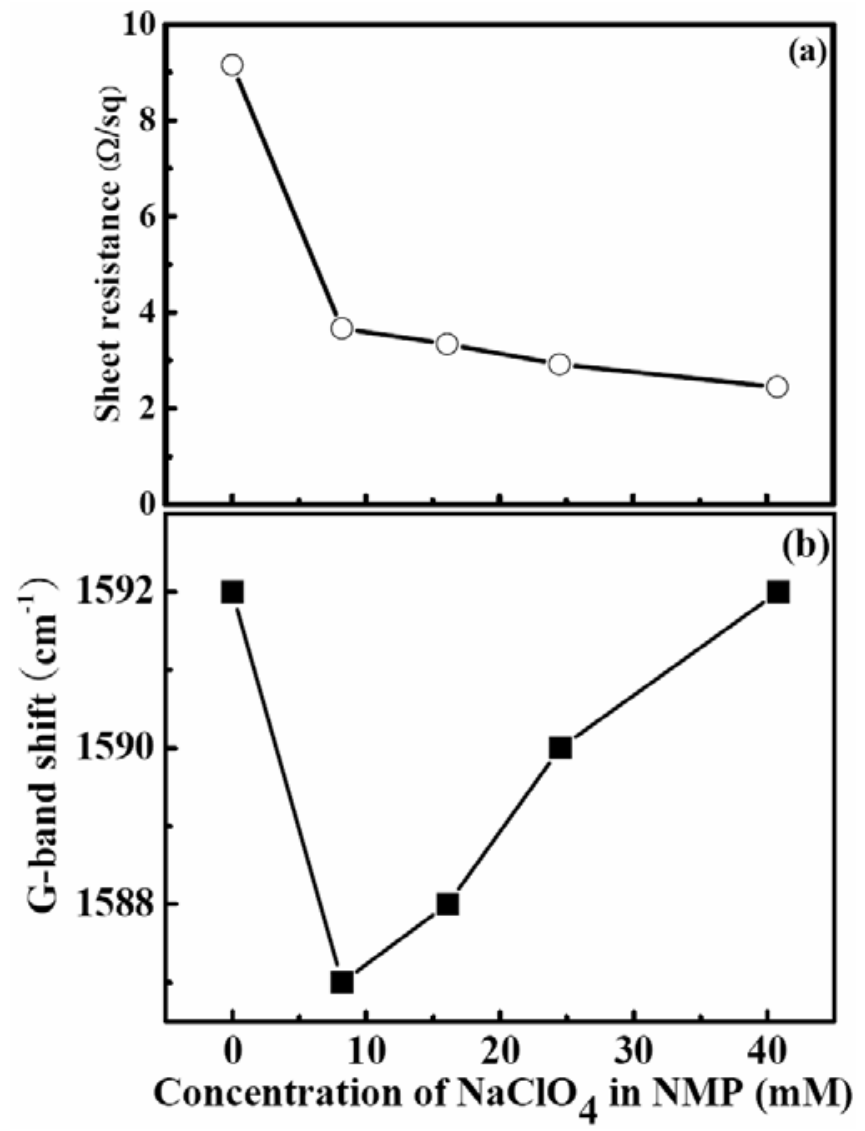

Figure S2: As a function of concentration of $\mathrm{NaClO}_{4}$, (a) sheet resistance and (b) G-band shift in Raman spectra at an excitation of $633 \mathrm{~nm}$. 


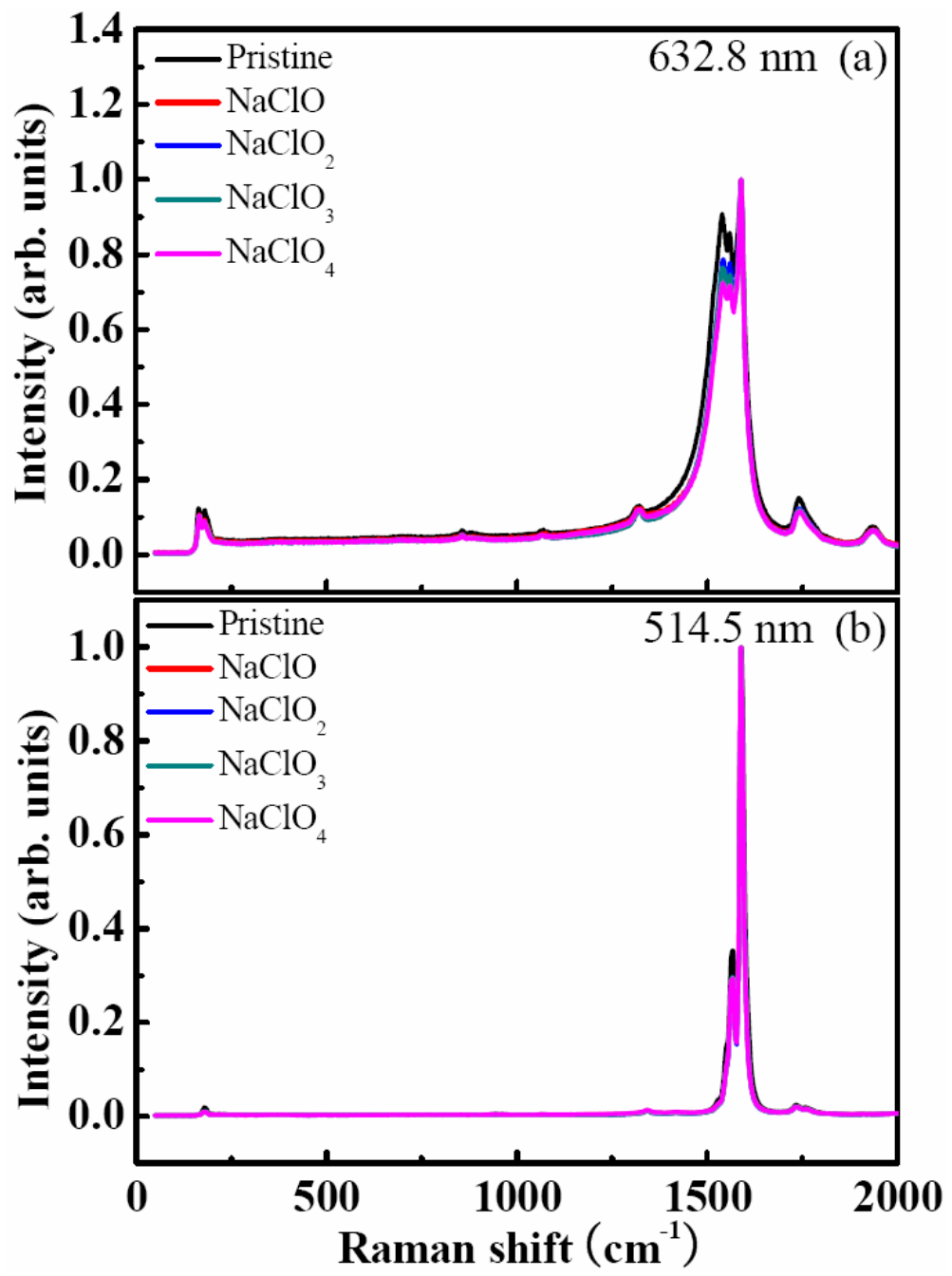

Figure S3: Raman spectra at an excitation of $633 \mathrm{~nm}$ and $514 \mathrm{~nm}$ of the pristine and chloro oxoanion treated samples at different oxidation numbers after heat treatment at $600{ }^{\circ} \mathrm{C}$ for 1 hour. The Raman spectra including the radial breathing modes, and G-band were recovered almost completely after heat treatment. 